\title{
Protocol for a Systematic Review of the Effectiveness of Provider-targeted Interventions to Improve Opioid Prescribing in Primary Care
}

\section{Pallavi Prathivadi ( $\sim$ pallavi.prathivadi@monash.edu)}

Monash University Faculty of Medicine Nursing and Health Sciences https://orcid.org/0000-00017909-6962

\section{Natalie Connell}

Stanford University School of Medicine

\section{Louisa Picco}

Monash University Faculty of Medicine Nursing and Health Sciences

\section{Karleen F Giannitrapani}

Stanford University School of Medicine

\section{Hong-nei Wong}

Stanford University School of Medicine

\section{Chris Barton}

Monash University Faculty of Medicine Nursing and Health Sciences

\section{Karl Lorenz}

Stanford University School of Medicine

\section{Danielle Mazza}

Monash Medical School: Monash University Faculty of Medicine Nursing and Health Sciences

\section{Protocol}

Keywords: Behaviour change interventions, narrative synthesis, opioid prescribing, prescribing, primary care provider, Theoretical Domains Framework

Posted Date: March 1st, 2021

DOl: https://doi.org/10.21203/rs.3.rs-266226/v1

License: (c) (i) This work is licensed under a Creative Commons Attribution 4.0 International License.

Read Full License 


\section{Abstract}

\section{Background:}

Improving primary care opioid prescribing is a public health priority in many western nations. Governments, policymakers and key stakeholders are intervening on multiple levels to address patient, prescriber and systems factors contributing to opioid over-prescription in primary care. Many opioid prescribing interventions specifically target primary care providers (PCPs); however, the overall effectiveness of these interventions is not known. Identifying effective components of PCP-targeted behaviour change interventions may help inform scalability and translation of prescribing interventions across countries and varying primary healthcare settings. The aim of this systematic review is to assess the effectiveness of provider-targeted interventions to improve opioid prescribing in primary care. This protocol reports the methods of the proposed narrative synthesis review that will be guided by the Theoretical Domains Framework (TDF).

\section{Methods:}

The study will follow Cochrane methods for conducting a narrative synthesis. Reporting is compliant with the Preferred Reporting Items for Systematic Reviews and Meta-analysis (PRISMA) protocols. The review will conduct searches in PubMed, EMBASE, PsychInfo, CINAHL, and the Cochrane Library databases for studies published in the English language from 2010 onwards. Reference lists of accepted articles will be also screened for additional studies meeting inclusion. Any opioid prescribing behaviour will be measured as an outcome. Intervention components will be mapped to domains of the TDF. No geographic limits will be applied. All stages of screening and data extraction will involve a dual review with gold standard adjudication. The Cochrane Risk of Bias tool will be used to evaluate quality and risk.

\section{Discussion:}

This review is being conducted in strict adherence to Cochrane principles. The protocol was submitted for registration to Prospero prior to publication for transparency and to avoid duplication of research. Formal ethics approval is not required for this research. The findings of this review will inform the delivery and implementation of PCP targeted opioid prescribing interventions. Findings will be disseminated to a wide range of stakeholders involved in quality improvement, prescribing interventions, education and training; professional groups, policymakers, researchers and PCPs.

\section{Systematic review registration:}

Submitted to Prospero 22 December 2020; pending registration

\section{Background}

Reducing opioid related morbidity and mortality is an increasing global public health priority [1]. Described colloquially as the 'opioid crisis', the over-prescription of pharmaceutical opioids has caused 
significant, ongoing health impacts in many western nations, including USA, UK and Australia [2]. Despite only weak evidence supporting the therapeutic benefit of opioids in most chronic pain conditions, almost half of all opioid prescriptions in Australia are prescribed to manage chronic or long term pain $[3,4]$. In the USA, similarly, approximately $50 \%$ of pharmaceutical opioids are prescribed for periods of over 6 months and for chronic conditions $[5,6]$. As a result, governments, policymakers and key stakeholders are intervening on multiple levels to address patient, prescriber and systems factors contributing to opioid over-prescription [1].

Efforts to reduce opioid prescribing frequently target primary care providers (PCPs), who manage the bulk of chronic pain, and are the predominant prescribers of opioids [7, 8]. Mixed methods studies over the last decade show the opioid prescribing patterns of PCPs are multifactorial $[9,10]$. Providers' decisions to prescribe opioids- despite evidence that questions their use- may be influenced by many considerations including the unaffordable cost, or inaccessibility of non-pharmacological pain management, lack of suitable drug alternatives, perceived therapeutic benefit, insufficient appreciation of opioid harm, and misperception that opioids improve patients' quality of life $[8,11,12]$. However, the drivers of PCP opioid overprescribing vary markedly by country and setting; drug marketing, prescribing incentives, risk of illicit diversion, and pay-for-service healthcare models are also important socio-political factors [13]. In the USA, the rise of synthetic opioids has particularly contributed to opioid mortality in the last decade [14]. Despite this, similar interventions are described in the literature to improve primary care prescribing in various countries, without necessarily considering the context dependent factors which may be contributing to the local opioid prescribing practices.

Implementation scientists have long urged for primary care interventions to be evaluated for feasibility and acceptability across complex primary care settings worldwide $[15,16]$. The literature describes a wide range of interventions using well known behaviour change principles to encourage change in opioid prescribing practices $[17,18]$. These include guideline implementation and distribution of educational materials, educational meetings, local consensus processes, educational outreach, peer opinion leaders, audit and feedback, reminders, marketing, mass media, incentives and penalties [19]. Despite ongoing efforts to change prescribing, there is still no clear consensus about the effectiveness of these different behaviour change interventions in actually changing PCP behaviours. Furthermore, it is difficult to determine which factors contributing to opioid overprescribing these effective interventions actually target. Identifying relevant behaviour change approaches to PCP targeted interventions may also inform scalability and translation across countries and varying primary healthcare settings for future efforts.

\section{Aims and theoretical underpinning}

The primary aim of this systematic review is to assess the effectiveness of provider-targeted interventions to improve opioid prescribing in primary care. Our secondary aim is to identify the behaviour change mechanisms associated with these interventions and narratively explore the relevant behaviour change theoretical domains and constructs conceptualised using the Theoretical Domains Framework (TDF) (version 2) [20, 21]. The mapping of intervention components to TDF domains will be undertaken 
by the review team, as it is unlikely that study authors would have completed this theoretical exercise. Several members of the study team (PP, DM and CB) have experience and expertise in applying the TDF to mixed method research and will contact study authors if further information is needed.

Given the heterogeneity in opioid prescribing interventions, and the complex nature of these widely ranging interventions, our research questions will be best addressed through a narrative synthesis methodology. To the best of our knowledge, a similar review has not been undertaken before. This review will therefore address a gap in the current evidence relevant to PCPs, policymakers, pharmaceutical companies, providers of continuing medical education and other stakeholders in the issue of opioid overprescribing. This review will also contribute to existing literature about behaviour change theory by evaluating the application of behaviour change theory in the implementation of prescribing interventions.

\section{Methods/design}

This protocol is conducted and reported in compliance with the Preferred Reporting Items for Systematic Reviews (PRISMA) guidelines [23]. The review team is a multidisciplinary group of practicing primary care providers (family medicine and palliative care), primary care and pain management researchers, medical subject librarian and early career medical researchers. A collaborative and experienced approach has been taken to develop the research questions and methodology, guided by the Participant-InterventionComparator-Outcome (PICO) format.

\section{Objectives}

The objective of the proposed review is to assess the effectiveness of primary care provider-targeted interventions to improve opioid prescribing in primary care by addressing three research questions relating to intervention effectiveness:

a. What types of primary care provider-targeted interventions successfully change opioid prescribing behaviours?

b. What types of opioid prescribing behaviours or practices do these interventions seek to change?

c. How do the behaviour change mechanisms of these interventions differ, and what factors are associated with successful change in opioid prescribing practices?

\section{Study design}

The study will follow Cochrane methods for conducting a narrative synthesis [24]. This methodology has been chosen as it will most appropriately assess the wide range of complex interventions that we identified in our earlier scoping. The pilot search of this review has shown that meta-analysis cannot be undertaken due to heterogeneity of the interventions. This review will follow the four major steps for conducting narrative synthesis in reviews of intervention effectiveness: (1) Developing a theory of how and why the intervention works, and for whom; (2) Developing a preliminary synthesis of the included 
studies; (3) Exploring relationships in the data within and between studies; and (4) Assessing the robustness of the synthesis [24].

\section{Study setting}

This review studies the opioid prescribing behaviours of PCPs. Opioids may be prescribed for any chronic or acute pain condition, excluding palliative or end-of-life care. Studies eligible for inclusion will occur in an ambulatory primary care setting, target PCPs and measure change in one or more aspects of opioid prescribing as a consequence of the intervention. We will include randomised control trials that aim to change PCP opioid prescribing behaviours, including pilot studies if they meet inclusion criteria. Systematic reviews and meta-analyses will be disassembled for extraction of individual studies if eligibility criteria are met for the individual studies. Initial screening at the title and abstract stage demonstrated a low number of studies likely to proceed to full text screening. Therefore extracting individual studies from systematic review reference lists is a feasible method of increasing data to inform this review. Studies will be excluded if they are interventions targeting patients or carers, unless a component of the study includes changing provider behaviours, have the sole aim of evaluating drug effectiveness, are protocols, are non-randomised or lack a comparison group. Studies will also be excluded if they focus on prescribing by providers in emergency departments or hospital inpatient settings.

\section{Participants}

This review will include all studies of PCPs qualified to prescribe opioid medications to patients in an ambulatory primary care setting. Providers may include physicians, physician assistants, nurse practitioners and clinical pharmacists who are qualified to prescribe opioids to primary care patients.

\section{Interventions and comparisons}

Included studies will implement an intervention designed to change opioid prescribing. The intervention must target the PCP and seek to change prescribing behaviours. Interventions will be defined as professional or provider targeted and delivered directly to the PCPs to change behaviours. These interventions are defined using the taxonomy of behaviour change interventions for guideline implementation and include distribution of educational materials, educational meetings, local consensus processes, educational outreach, peer opinion leaders, audit and feedback, reminders, marketing, mass media, incentives and penalties and others which may be undefined. The main comparator is no intervention or no active efforts to change provider opioid prescribing practices. Studies with two intervention arms (and no control) will be included if each arm involves a distinctly different behaviour change approach.

\section{Outcomes}

The primary outcome of interest is opioid prescribing practices. For the purposes of this review, all outcomes will be collectively referred to as 'opioid prescribing behaviours' and will include actual or selfreported measurements of prescribing by PCPs. Outcome measurements may refer to rate, type and 
frequency of opioid prescription (including opioid agonist therapy), use of opioid risk reduction strategies, guideline concordance or opioid-related adverse events amongst others. The secondary outcome of interest is patient-related clinical outcomes. This will include actual or self-reported measurements by patients about the consequence of changing providers' opioid prescribing behaviours including prescription opioid use, illicit opioid use, pain severity, opioid-related adverse events and similar.

\section{Search strategy}

A preliminary search was undertaken in December 2020 using PubMed, Google Scholar, the Cochrane Library and PROSPERO to identify potential similar reviews, background literature and estimate the volume of published literature on this topic. The search strategy was developed considering the research questions and PICO criteria, and refined with assistance from the Stanford University medical subject librarian (Table 1). The review will conduct searches in PubMed, EMBASE, PsychInfo, CINAHL, and the Cochrane Library databases for studies published in the English language from 2010 onwards. Reference lists of accepted articles will be also screened for additional studies meeting inclusion. No geographic limits will be applied. 
Population ～("primary health care"[MH] OR "primary care"[tw] OR "primary health care"[tw] OR "primary healthcare"[tw] OR "physicians, primary care"[Mesh] OR "Primary care physicians"[tw] OR "primary care physician"[tw] OR "Generalist"[tw] OR "General practitioners"[MH] OR "general practitioners"[tw] OR "general practitioner"[tw] OR "General practice"[MH] OR "general practice"[tw] OR "general practices"[tw] OR "general medicine"[tw] OR "family medicine"[tw] OR "family practice"[MH] OR "family practices" [tw] OR "family practice"[tw] OR "Primary care clinic"[tw] OR "Family health Clinic"[tw] OR "Family medicine clinic"[tw] OR "Primary care clinics"[tw] OR "Family health Clinics" [tw] OR "Family medicine clinics"[tw] OR "family physician*"[tw] OR "general medicine clinic"[tw] OR "general medicine clinics"[tw] OR "primary care nursing"[MH] OR "physicians, primary care"[MH] OR ("women's health center*"[TW] OR "woman's health center*"[TW] OR "women's health clinic*"[TW] OR "women's health facilit*"[TW] OR "women's health services"[MH] OR "women health service*"[TW] OR "woman health service*"[TW] OR "women's health service*"[TW] OR "women's health care service*" OR "women's health"[MH] OR "women's health practice*"[TW] OR "women's health"[MH] OR "women' healthcare"[TW] OR "women's health"[TW] OR "woman health"[TW] OR "woman's health"[TW] OR ("Community pharmacy services"[MeSH] OR "Pharmaceutical services" [MeSH] OR pharmacist*[All Fields] OR pharmacists[Mesh] OR "nurse practitioners"[Mesh] OR "nurse practitioner*" OR "advanced practice nursing"[Mesh] OR "advanced practice nurs*" OR "advance practice nurs*" OR "doctor of nursing practi*") ))

Intervention ("systematic review*"[tw] OR "systematic review"[pt] OR "meta-analysis"[pt] OR "metaanalys*"[tw] OR "metaanalys*"[tw] OR medline[tw] OR Cochrane[tw] OR Embase[tw] OR CINAHL[tw] OR (database*[tiab] AND search*[tiab]) OR

"randomized controlled trial"[PT] OR "controlled clinical trial"[PT] OR "randomiz*"[tiab] OR "placebo"[tiab] OR "clinical trials as topic"[MH] OR "randomly"[tiab] OR "trial"[ti] OR "clinical trial, phase III"[pt] OR "phase 3"[tw] OR "phase3"[tw] OR "phase III"[TW] OR "P3" [TW] OR "PIII"[TW] OR "random allocation"[MH] OR "double-blind method"[MH] OR "single-blind method"[MH] OR "clinical trial"[pt] OR "clinical trial, phase I"[pt] OR "clinical trial, phase II"[pt] OR "clinical trial, phase IV"[pt] OR "multicenter study"[PT] OR "clinical trial*"[tw] OR placebo*[tw] OR sham[tiab] OR "pilot"[tiab] OR "pilots"[tiab] OR "pilot studies"[tiab] OR "pilot study"[tiab] OR "pilot project"[tiab] OR "pilot projects"[tiab] OR "pilot projects"[MH])

Outcome Opioid* OR opiate OR "analgesics, opioid"[Mesh] OR "narcotics"[Mesh] OR "narcotic" [tw] OR "opiate alkaloids"[Mesh]) AND (prescrib*[tw] OR "prescription*" [tw] OR prescriber OR "drug prescriptions"[Mesh] OR "prescriptions"[Mesh] OR "inappropriate prescribing"[Mesh] OR "practice patterns, physicians"[Mesh] OR "guideline adherence" [Mesh] OR (guideline[tiab] AND (adher*[tiab] OR concordance[tw] OR compliance[tw] OR comply[tw])) OR "Opioid-Related Disorders"[mesh] OR "Prescription Drug Misuse" [mesh] OR "drug utilization"[Mesh])

Limits and restrictions

"English"[la] NOT ("animals"[MH] NOT "humans"[MH]) NOT ("letter"[PT] OR "case reports"[pt] OR "editorial"[pt] OR "comment"[PT] OR "protocol"[TI] OR "technical note"[TI] OR "technical notes"[TI] OR "technical report"[TI] OR "technical review"[TI] OR "technical solution"[TI]) AND 2010:2021[DP]

\section{Data extraction and synthesis}

All data will be managed using Covidence systematic review management system. Titles and abstracts of articles returned from initial searches will be dual screened based on the eligibility criteria outlined 
previously. Following this, full texts will be examined in detail and dual screened for eligibility. Conference proceedings and abstracts without full text will be excluded. Any disagreements will be resolved by a third-party adjudicator (DM or KL). All data will be extracted independently by two reviewers (PP, and either KG, LP or NC) using a customised data extraction form. This will be used to extract relevant data related to methodological issues, interventions, and opioid prescribing outcomes.

We will perform this process by identifying the key attributes of each intervention targeting PCPs, and through thematic synthesis using the TDF to identify which behaviour change mechanisms are being used and associated with effective change in opioid prescribing behaviours. If we are unable to assess this information in the published text, corresponding authors will be contacted for clarification and additional information.

Because we will include studies of any quality, we will perform sensitivity analyses to see how the data changes when including only high-quality randomised control trials or systematic review studies. The Cochrane Risk of Bias tool will be used to evaluate risk at outcome and study level [25]. All studies will undergo a dual review with gold standard adjudication.

\section{Discussion}

This review is being conducted in strict adherence to Cochrane principles. The protocol was submitted for registration on PROSPERO prior to publication for transparency, to avoid duplication of research and for real-time amendments or changes to the methodology. Formal ethics approval is not required for this research as there is no involvement of participants and the review is an analysis of publicly available studies. The main practical issue in conducting this review is clarity of key terms (prescribing intervention and opioid prescribing practices) and cohesively synthesising findings from these terms. The study team comprises experts in systematic reviews, opioid researchers and implementation scientists, who will work closely to ensure rigour in methodology and consensus at each stage of the review. However, the geographical spread of the research team limits their ability to meet in person. Similar to the process used in a previous review by this team [26], we will meet weekly through online platforms to ensure timely and practical progress of the research.

The review will be undertaken as part of a doctoral body of research (PP) studying primary care opioid prescribing. The findings of this review will inform the design and delivery of a PCP targeted complex intervention to change opioid prescribing, which will be undertaken by the same research team. Findings will be disseminated to a wide range of stakeholders involved in quality improvement, prescribing interventions, education and training; professional groups, policymakers, researchers and PCPs. These audiences are well placed to directly influence policy and practice and broker rapid translation of the review's findings. Additionally, the outcomes of the review will be published and presented after completion.

\section{List Of Abbreviations}


PCP Primary Care Provider

PICO Participant-Intervention-Comparison-Outcome

PRISMA Preferred Reporting Items for Systematic Reviews and Meta-Analyses

TDF Theoretical Domains Framework

UK United Kingdom

USA United States of America

\section{Declarations}

\section{Ethics approval and consent to participate}

Not applicable

Consent for publication

Not applicable

\section{Availability of data and materials}

The datasets used and/or analysed during the current study are available from the corresponding author on reasonable request.

\section{Competing interests}

The authors declare that they have no competing interests

\section{Funding}

Not applicable

\section{Authors' contributions}

DM and $\mathrm{KL}$ guided development of the review methodology, research question and search strategy. NC, LP, KG and PP developed the PICO, refined the research question, undertook scoping of pre-existing literature and undertook a pilot search. HW developed the search strategy and performed the review search. DM and CB supported PP to identify relevant theoretical approaches used in the review. PP was a major contributor in writing the manuscript and supported by all co-authors. All authors read and approved the final manuscript.

\section{Acknowledgements}




\section{References}

1. Degenhardt, L., et al., Global patterns of opioid use and dependence: harms to populations, interventions, and future action. The Lancet, 2019. 394(10208): p. 1560-1579.

2. Nolan, S., M.E. Socias, and E. Wood, The threat of an international opioid crisis. Current Addiction Reports, 2018. 5(4): p. 473-477.

3. Noble, M., et al., Long-term opioid management for chronic noncancer pain. Cochrane database of systematic reviews, 2010(1).

4. Lalic, S., et al., Prevalence and incidence of prescription opioid analgesic use in Australia. British journal of clinical pharmacology, 2019. 85(1): p. 202-215.

5. Volkow, N.D. and A.T. McLellan, Opioid abuse in chronic pain-misconceptions and mitigation strategies. New England Journal of Medicine, 2016. 374(13): p. 1253-1263.

6. Sullivan, M.D., et al., Trends in use of opioids for non-cancer pain conditions 2000-2005 in commercial and Medicaid insurance plans: the TROUP study. Pain, 2008. 138(2): p. 440-449.

7. Bedson, J., et al., Trends in long-term opioid prescribing in primary care patients with musculoskeletal conditions: an observational database study. Pain, 2016. 157(7): p. 1525.

8. Tong, S.T., et al., Chronic opioid prescribing in primary care: factors and perspectives. The Annals of Family Medicine, 2019. 17(3): p. 200-206.

9. Richards, G.C., et al., Factors associated with the prescribing of high-dose opioids in primary care: a systematic review and meta-analysis. BMC medicine, 2020. 18: p. 1-11.

10. Lozada, M.J., et al., Opioid Prescribing by Primary Care Providers: a Cross-Sectional Analysis of Nurse Practitioner, Physician Assistant, and Physician Prescribing Patterns. Journal of general internal medicine, 2020. 35(9): p. 2584-2592.

11. Prathivadi, P., C. Barton, and D. Mazza, Qualitative insights into the opioid prescribing practices of Australian GP. Family practice, 2020. 37(3): p. 412-417.

12. Jamison, R.N., et al., Beliefs and attitudes about opioid prescribing and chronic pain management: survey of primary care providers. Journal of opioid management, 2014. 10(6): p. 375-382.

13. Dasgupta, N., L. Beletsky, and D. Ciccarone, Opioid crisis: no easy fix to its social and economic determinants. American journal of public health, 2018. 108(2): p. 182-186.

14. Mattson, C.L., et al., Annual surveillance report of drug-related risks and outcomes-United States, 2017. 2017.

15. Wensing, M., R. Grol, and J. Grimshaw, Improving patient care: the implementation of change in health care. 2020: Wiley-Blackwell.

16. Greenhalgh, T., How to implement evidence-based healthcare. 2017: John Wiley \& Sons. 
17. Johnson, M.J. and C.R. May, Promoting professional behaviour change in healthcare: what interventions work, and why? A theory-led overview of systematic reviews. BMJ open, 2015. 5(9): p. e008592.

18. Michie, S., et al., From theory to intervention: mapping theoretically derived behavioural determinants to behaviour change techniques. Applied psychology, 2008. 57(4): p. 660-680.

19. Mazza, D., et al., Refining a taxonomy for guideline implementation: results of an exercise in abstract classification. Implementation Science, 2013. 8(1): p. 1-10.

20. Michie, S., et al., Making psychological theory useful for implementing evidence based practice: a consensus approach. BMJ Quality \& Safety, 2005. 14(1): p. 26-33.

21. Atkins, L., et al., A guide to using the Theoretical Domains Framework of behaviour change to investigate implementation problems. Implementation Science, 2017. 12(1): p. 77.

22. Pawson, R., et al., Realist review-a new method of systematic review designed for complex policy interventions. Journal of health services research \& policy, 2005. 10(1_suppl): p. 21-34.

23. Page, M.J., et al., Updating guidance for reporting systematic reviews: development of the PRISMA 2020 statement. 2020.

24. R, R., Cochrane Consumers and Communication Review Group: data synthesis and analysis', C.C.a.C.R. Group, Editor. 2013: http://cccrg.cochrane.org.

25. Higgins, J.P., et al., The Cochrane Collaboration's tool for assessing risk of bias in randomised trials. Bmj, 2011. 343: p. d5928.

26. Karleen Giannitrapani, K.L., Pallavi Prathivadi, Connie Wong, Natalie Connell, Sophia Zupanc, $A$ systematic review of studies of the effectiveness of primary care teams, teamwork and teaming to improve chronic pain management. PROSPERO, 2020.

\section{Supplementary Files}

This is a list of supplementary files associated with this preprint. Click to download.

- PRISMAPchecklist.docx 